\title{
Efecto de la aplicación precosecha de giberelinas y calcio en la producción, calidad y rajado del fruto de uchuva (Physalis peruviana L.)
}

\section{Effect of preharvest application of gibberellins and calcium on production, fruit quality and cracking of cape gooseberry (Physalis peruviana L.)}

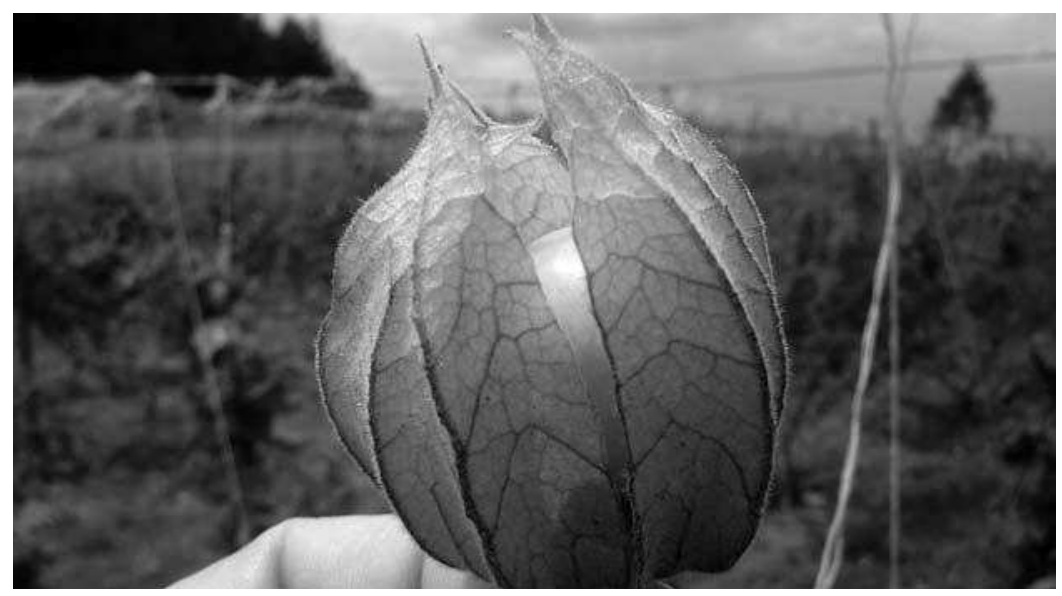

NATHALY AMÉZZUITA ${ }^{1}$

HELBER ENRIQUE BALAGUERA-LÓPEZ' JAVIER GIOVANNI ÁLVAREZ-HERRERA1, 2

Fruto de uchuva en su punto óptimo de cosecha.

Foto: G. Fischer

\section{RESUMEN}

La uchuva actualmente es la fruta exótica de mayor exportación en Colombia. Uno de los principales problemas fisiológicos que afecta la calidad y la rentabilidad del cultivo de uchuva es el rajado del fruto, además, se hace necesario aumentar la productividad y la calidad del fruto para hacerlo más competitivo en el mercado. Por tanto, en un diseño completamente al azar se evaluó el efecto de la aplicación en precosecha vía foliar de siete tratamientos correspondientes a diferentes concentraciones de giberelinas $\left(5,10\right.$ ó $\left.15 \mathrm{mg} \mathrm{L}^{-1}\right)$ y de calcio $\left(0,5 ; 1,0\right.$ ó $\left.1,5 \mathrm{~g} \mathrm{~L}^{-1}\right)$ y un testigo absoluto sobre la producción, calidad y rajado del fruto de uchuva. Las aplicaciones de los tratamientos se realizaron a los 3, 10, 17 y 24 días después de antesis. Las aplicaciones foliares de calcio y giberelinas aumentaron significativamente la producción total de uchuva, pero no hubo diferencias en la calidad extra y calidad I. Se presentó menor porcentaje de rajado de frutos con $10 \mathrm{mg} \mathrm{L}^{-1} \mathrm{de}$ giberelinas, seguido del tratamiento de $0,5 \mathrm{~g} \mathrm{~L}^{-1}$ de calcio. La aplicación de $0,5 \mathrm{~g} \mathrm{~L}^{-1}$ de calcio generó mayor firmeza y contenido de sólidos solubles totales en los frutos, mientras que todos los tratamientos fueron estadísticamente superiores al testigo en la acidez total titulable e inferiores en la relación de madurez, esto indica que las giberelinas y el calcio retrazan la madurez de los frutos de uchuva y potencialmente pueden tener mayor vida en poscosecha.

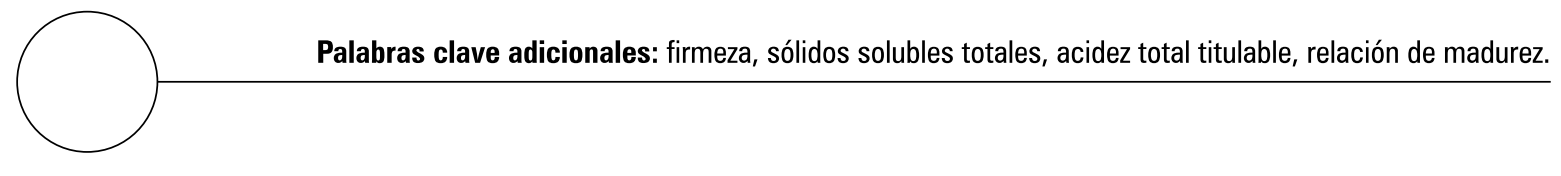

Grupo de Investigaciones Agrícolas, Facultad de Ciencias Agropecuarias, Universidad Pedagógica y Tecnológica de Colombia, Tunja (Colombia).

2 Autor para correspondencia. jgalvarezh@gmail.com 


\section{ABSTRACT}

Cape gooseberry is currently the main exotic fruit of export in Colombia. Fruit cracking is one of the main physiological problems that affect the quality and rentability of cape gooseberry crop; also it is necessary to increase the productivity and fruit quality to make this plant more competitive in the market. Therefore, in a completely randomized design, the effect of preharvest foliar application of seven treatments corresponding to different concentrations of gibberellins $\left(5,10\right.$ or $\left.15 \mathrm{mg} \mathrm{L}^{-1}\right)$ and calcium $\left(0.5,1.0\right.$ or $\left.1.5 \mathrm{~g} \mathrm{~L}^{-1}\right)$ and a control was evaluated on the production, quality and fruit cracking of cape gooseberry. The applications of treatments were done at $3,10,17$ and 24 days after anthesis. Foliar applications of calcium and gibberellins significantly increased the total production of cape gooseberry, but no differences in the quality extra and quality I were observed. It was presented a low percentage of fruit cracking with $10 \mathrm{mg} \mathrm{L}^{-1}$ gibberellins followed by treatment of $0.5 \mathrm{~g} \mathrm{~L}^{-1}$ calcium. The application of $0.5 \mathrm{~g} \mathrm{~L}^{-1}$ calcium generated higher firmness and higher soluble solids content in fruit, while all treatments were statistically higher than control in the total acidity and lower in terms of maturity ratio. This suggests that gibberellins and calcium delay the maturity of the fruits of cape gooseberry and potentially contribute longer post-harvest life.

Additional key words: firmness, total soluble solids, total titratable acidity, maturity ratio.

La uchuva (Physalis peruviana L.) es la fruta exótica de mayor exportación en Colombia. Para el 2008, el país contó con una producción de 15.463 t, 841 ha cosechadas y una tasa de crecimiento anual de $42,6 \%$ y $33,7 \%$ para la producción y el área cosechada respectivamente, donde los departamentos de mayor producción en orden fueron: Boyacá, Cundinamarca y Antioquia (Agronet, 2008). Estas importantes cifras justifican la necesidad de continuar mejorando el nivel tecnológico del cultivo para aumentar la producción, la productividad, la calidad del fruto $y$, por ende, la rentabilidad del cultivo, todo esto mediante la investigación y desarrollo de nuevas técnicas de manejo.

Uno de los principales problemas fisiológicos que afectan la calidad y la rentabilidad del cultivo de uchuva es el rajado de los frutos. Esta anomalía hace que en cosecha y en poscosecha se pierda más de $30 \%$ de la producción (Gordillo et al.,
2004) y hasta $50 \%$ de los frutos son rechazados por el exportador en la época de lluvia (Fischer, 2005), lo que arroja pérdidas tanto para los productores de fruta como para los comercializadores y consumidores.

El rajado de los frutos, se debe a una serie de factores intrínsecos y extrínsecos. Aunque el mecanismo por el cual se genera el rajado no es completamente conocido, se ha sugerido que es un fenómeno osmótico (Sekse, 1995; Pratella, 1996), acompañado por pérdida de la integridad de las membranas celulares; por consiguiente, concentraciones de calcio que generen mayor firmeza en el fruto, contribuirán a disminuir el rajado. El rajado también puede darse debido a que el parénquima de los frutos de uchuva no es compacto y presenta numerosas "lagunas", cuyo número y tamaño aumenta a medida que el fruto madura (Valencia, 1985); el grado de cohesión celular está directamente relacionado con 
la capacidad de retención de líquidos dentro de la pulpa de los frutos $y$, por tanto, con las fuerzas de presión en el interior de los mismos (Opara, 1997). Del mismo modo, los rendimientos potenciales del cultivo y la calidad en la cosecha se ven disminuidas por dosis inadecuadas de $\mathrm{Ca} \mathrm{y}$ boro (Cooman et al., 2005).

En tomate, se deben evitar cambios bruscos de humedad en el suelo durante la época de cosecha, ya que si esto ocurre, se presentan rajaduras en los frutos (Peet, 1992). Los incrementos súbitos en la humedad del suelo disminuyen la fuerza de tensión normal de la epidermis del fruto, reduciendo su fuerza de elasticidad y haciendo que ésta se agrande rápidamente y desarrolle el rajado (Gordillo et al., 2004). Sin embargo, mediante la aplicación foliar de calcio se logra aumentar la rigidez celular y con giberelinas es posible incrementar la extensibilidad y elasticidad de la célula, así como expresar una modificación de la estructura de la pared celular (Podestá et al., 2001), factores que pueden amortiguar el efecto negativo de los cambios bruscos de humedad en el suelo y disminuir el rajado del fruto de uchuva.

El Ca está implicado en varias funciones importantes dentro de la planta, principalmente en la estabilización y rigidez de membranas y paredes celulares (Marschner, 2002). Esto se da porque en la pared celular el calcio forma enlaces con los grupos carboxilos en cadenas de poligalacturonanos adyacentes presentes en la lámina media de las paredes celulares, esto contribuye a la adhesión y cohesión célula-célula (Hernández-Muñoz et al., 2008). A nivel de membranas, el calcio genera estabilidad al formar enlaces con los fosfolípidos y proteínas mediante los puentes fosfatos y carboxílicos, preferencialmente en la superficie de membranas (Marschner, 2002).

El calcio, por su capacidad para disminuir la permeabilidad hidráulica de las membranas celulares, reducir la absorción de agua y aumentar la firmeza de la pulpa en algunas especies (Brown et al., 1996), es el factor nutricional más estudia- do respecto al rajado de los frutos (Huang et al., 2005). Brown et al. (1996) proponen que la deficiencia de calcio está asociada con el rajado en cerezas, sin embargo, Podestá et al. (2001) encontraron que su aplicación precosecha no aumentó la resistencia al rajado. Respecto a la aplicación foliar de calcio, fue reportado que reduce el rajado en manzanas, duraznos, naranjas, peras, ciruelas e higos, mientras que hay reportes que no muestran efecto significativo en melón, uva y cerezas (Xu-Ming et al., 2008).

Las giberelinas están asociadas, entre otros, procesos, con la división y elongación celular (Taiz y Zeiger, 2006), la iniciación floral, desarrollo de los órganos florales, desarrollo de la fructificación e inducción de algunas enzimas hidrolíticas (Matsuoka, 2003). Según Taiz y Zeiger (2006), las giberelinas pueden favorecer el cuajado y crecimiento de algunos frutos como manzanas, además promueven el crecimiento celular debido a que incrementan la hidrólisis de almidón, fructosa y sacarosa, con lo que se originan moléculas de fructosa y glucosa, que contribuyen a la formación de la pared celular. La información sobre la acción de las giberelinas en el rajado de frutos no es consistente, algunos resultados indican que disminuyó el rajado (Looney y Lidster, 1980); sin embargo, Cline (1995, citado por Sansavini y Lugli, 1997) señala que no tuvo acción eficaz sobre este desorden fisiológico.

Para aumentar el tamaño de los frutos se han utilizado comercialmente aplicaciones foliares de ácido giberélico (Facteau et al., 1992). También se ha encontrado que esta hormona retrasa la maduración, aumenta la firmeza de la pulpa (Looney y Lidster, 1980; Facteau et al., 1992) e incrementa del contenido de sólidos solubles (Facteau et al., 1992).

Peet (1992) constata que todas las prácticas culturales que producen uniformidad y crecimiento relativamente lento ofrecen alguna protección contra el rajado del fruto. Esto significa que se deben evitar prácticas culturales que aumenten en 
exceso el crecimiento del fruto, sea un riego o una fertilización demasiado abundante o una aplicación de hormonas de crecimiento no adecuada.

Por tanto, el objetivo de esta investigación fue evaluar el efecto de la aplicación foliar en precosecha de diferentes concentraciones de giberelinas y calcio sobre la producción, calidad y rajado de frutos de uchuva.

\section{MATERIALES Y MÉTODOS}

El experimento se realizó en una plantación comercial de uchuva en la finca El Saque, localizada en la Vereda San Antonio, en el municipio de Tuta, Boyacá, que se encuentra a $2.600 \mathrm{msnm}$, 5०41'36" N y 73¹3'51' W. Este municipio cuenta con una temperatura promedio de $14^{\circ} \mathrm{C}$, humedad relativa del $80 \%$ y una precipitación promedio anual de $935 \mathrm{~mm}$.

Plantas de la Physalis peruviana, ecotipo Colombia, se sembraron con distancias de $2 \times 2 \mathrm{~m}$, en sistema de espaldera, conducidas en vaso abierto. A los $80 \mathrm{~d}$ después de la siembra, una vez las flores entraron en antesis, se seleccionaron las plantas a utilizar, las cuales presentaron condiciones homogéneas en función del porte, diámetro de tronco y altura de planta. A estas plantas se les realizaron las labores culturales típicas para la zona, el riego se suministró mediante goteo y la fertilización edáfica se hizo con base en el resultado del análisis de suelos (tabla 1). Al momento de la siembra se aplicó $200 \mathrm{~g} / \mathrm{planta}$ del la fórmula 10-20-20 + 30 g/planta de Nutrimins (N 8\%; $\mathrm{P}_{2} \mathrm{O}_{5} 5 \%$; $\mathrm{CaO} 18 \% ; \mathrm{MgO} 6 \%$; $1,6 \%$; Cu 0,14\%; B 1\%; Mo 0,005\% y Zn 2,5\%) + 50 g/planta de micorrizas $+2 \mathrm{~kg} /$ planta de materia orgánica (Abimgra). A los 6 meses se fertilizó en corona con $100 \mathrm{~g} /$ planta de 15-15-15 + 50 g/planta de Nitrabor (N 15,4\%; CaO 25,6\%; B 0,3\%) y 50 g/ planta de Nutrimins.

Se utilizó un diseño completamente al azar con siete tratamientos correspondientes a la aplica-

\section{Tabla 1. Propiedades fisicoquímicas del suelo antes} de la siembra.

\begin{tabular}{|l|r|l|}
\hline $\begin{array}{c}\text { Propiedad } \\
\mathrm{pH}\end{array}$ & Valor & \multicolumn{1}{|c|}{ Unidades } \\
\hline $\mathrm{Ca}$ & 5,62 & \\
\hline $\mathrm{Mg}$ & 0,22 & $\mathrm{cmol} \mathrm{kg}^{-1}$ de suelo \\
\hline $\mathrm{K}$ & 0,46 & $\mathrm{cmol} \mathrm{kg}^{-1}$ de suelo \\
\hline $\mathrm{Na}$ & 0,26 & $\mathrm{cmol} \mathrm{kg}^{-1}$ de suelo \\
\hline $\mathrm{P}$ & 141,90 & $\mathrm{mg} \mathrm{kg}^{-1}$ de suelo \\
\hline $\mathrm{Fe}$ & 431,63 & $\mathrm{mg} \mathrm{kg}^{-1}$ de suelo \\
\hline $\mathrm{Cu}$ & 0,88 & $\mathrm{mg} \mathrm{kg}^{-1}$ de suelo \\
\hline $\mathrm{Zn}$ & 2,35 & $\mathrm{mg} \mathrm{kg}^{-1}$ de suelo \\
\hline $\mathrm{Mn}$ & 2,96 & $\mathrm{mg} \mathrm{kg}^{-1}$ de suelo \\
\hline $\mathrm{S}$ & 19,40 & $\mathrm{mg} \mathrm{kg}^{-1}$ de suelo \\
\hline $\mathrm{B}$ & 0,56 & $\mathrm{mg} \mathrm{kg}^{-1}$ de suelo \\
\hline $\mathrm{CIC}$ & 7,27 & $\mathrm{cmol} \mathrm{kg}^{-1}$ de suelo \\
\hline $\mathrm{CE}$ & 0,25 & $\mathrm{dS} \mathrm{m}^{-1}$ \\
\hline
\end{tabular}

ción de cuatro concentraciones de giberelinas $\left(\mathrm{GA}_{3}\right)\left(5,10\right.$ ó $\left.15 \mathrm{mg} \mathrm{L}^{-1}\right)$ y cuatro concentraciones de calcio $\left(0,5 ; 1,0\right.$ ó $\left.1,5 \mathrm{~g} \mathrm{~L}^{-1}\right)$ y un testigo absoluto sin aplicación. Cada tratamiento tuvo tres repeticiones, para un total de 21 unidades experimentales (UE). Cada UE estuvo compuesta por tres plantas de uchuva. Los tratamientos fueron aplicados vía foliar con fumigadora de espalda de $20 \mathrm{~L}$ a los 3, 10, 17 y 24 d después de antesis. La fuente de calcio utilizada fue $\mathrm{Ca}\left(\mathrm{NO}_{3}\right)_{2}$, mientras que para las giberelinas se utilizó ácido giberélico al 10\% (Progibb, Bayer CropScience, Bogotá).

La cosecha de los frutos de uchuva se llevó a cabo en el punto de madurez recomendado por la Norma Técnica Colombiana NTC 4580 (Icontec, 1999): color 5 y 6 (frutos de color anaranjado y anaranjado intenso). Una vez realizada la cosecha se midieron las siguientes variables: (1) Producción por calidades comerciales ( $\mathrm{kg} \mathrm{ha}^{-1}$ ) de $\mathrm{c} / \mathrm{u}$ de las calidades comerciales según norma NTC 4580, calidad extra, calidad I y calidad II; (2) Producción $\left(\mathrm{kg} \mathrm{ha}^{-1}\right)$ como sumatoria de la producción obtenida en $\mathrm{c} / \mathrm{u}$ de las calidades comerciales; (3) Frutos rajados (\%): se calculó el porcentaje con base en el peso de todos los frutos 
que presentaron los síntomas del rajado durante la cosecha por cada calidad comercial.

Se realizó la evaluación de las variables de calidad de fruto, estas fueron: (1) Acidez total titulable (ATT) mediante cálculos con datos de volumen de $\mathrm{NaOH}$, incorporado en $5 \mathrm{~g}$ de jugo de frutos agregando tres gotas de fenoftaleína en una titulación potenciométrica hasta $\mathrm{pH}$ de 8,2; (2) Sólidos solubles totales (SST) a través de mediciones de grados Brix con un refractómetro manual marca Atago; (3) Relación de madurez (RM) se determinó mediante la relación entre los SST y la ATT; (4) Firmeza del fruto (N) mediante la utilización de un penetrómetro digital PCE-PTR200.

Con los datos obtenidos se realizó un análisis de varianza y se hizo una prueba de comparación de promedios de Tukey al 5\%. Para el análisis de los datos se utilizó el programa SAS v. 8 e.

\section{RESULTADOS Y DISCUSIÓN}

\section{Producción}

La calidad extra no presentó diferencias estadísticas entre tratamientos, sin embargo, el mayor rendimiento se obtuvo con $10 \mathrm{mg} \mathrm{L}^{-1}$ de giberelinas seguido de $1,5 \mathrm{~g} \mathrm{~L}^{-1}$ de calcio y la menor respuesta se logró con el testigo. Respecto a la calidad I, tampoco se presentaron diferencias estadísticas, pero hubo mayor producción con $5 \mathrm{mg} \mathrm{L}^{-1}$ de giberelinas. Por su parte, la calidad II fue significativamente diferente entre tratamientos, se obtuvo mayor cantidad de frutos con $1,5 \mathrm{~g} \mathrm{~L}^{-1}$ de calcio y menor respuesta con el testigo. Para el rendimiento total, con diferencias estadísticas el mejor tratamiento fue la aplicación de 1,5 $\mathrm{g} \mathrm{L}^{-1}$ de calcio con $4.482,59 \mathrm{~kg} \mathrm{ha}^{-1}$, a continuación le siguió el tratamiento de $5 \mathrm{mg} \mathrm{L}^{-1}$ de giberelinas (4.402,98 $\mathrm{kg} \mathrm{ha}^{-1}$ ) y la menor producción fue obtenida con el testigo absoluto $\left(3.578,93 \mathrm{~kg} \mathrm{ha}^{-1}\right)$ (figura 1).

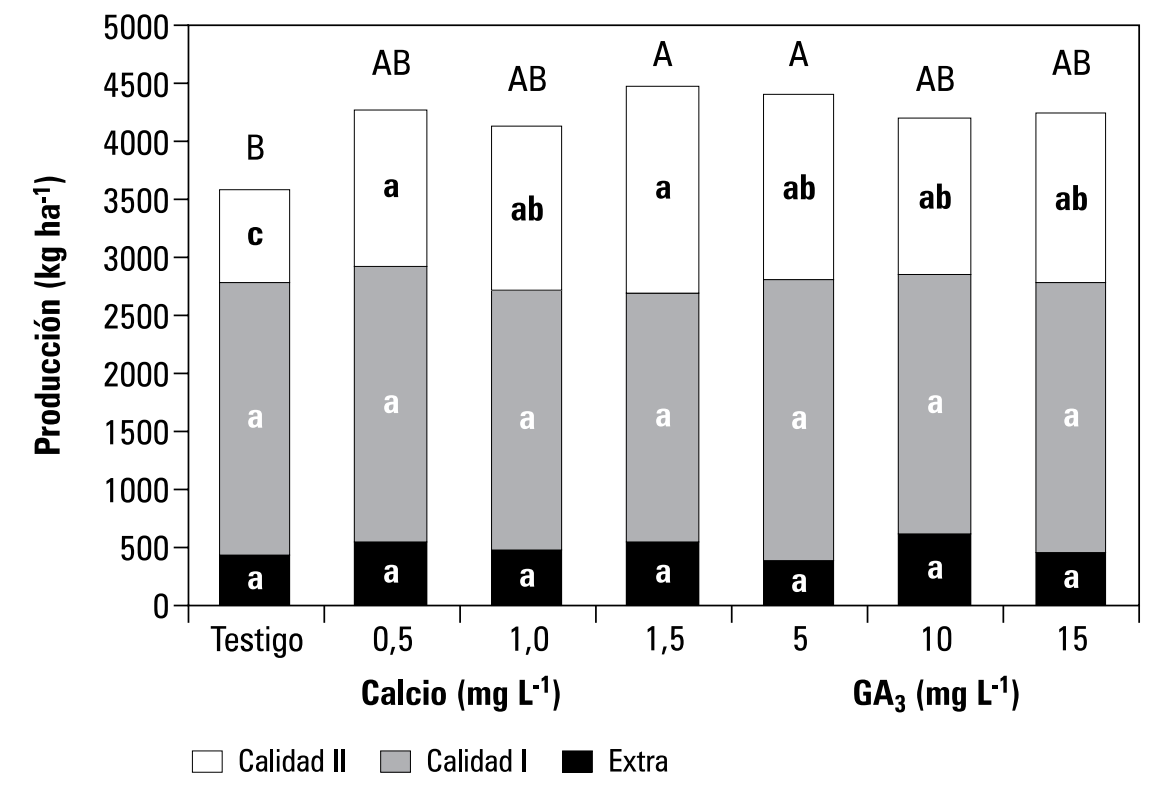

Figura 1. Efecto de las aplicaciones foliares de calcio y giberelinas en precosecha sobre la producción de uchuva por calidades comerciales. Promedios con letras distintas en la misma serie indican diferencias significativas según la prueba de Tukey $(P \leq 0,05)$. 
Los resultados indican que el calcio y las giberelinas influyen sobre la producción de frutos de uchuva. El calcio aumentó el rendimiento debido posiblemente a que es un elemento esencial para las plantas, indispensable para la formación del tubo polínico (Barker y Pilbeam, 2007), constituye la lámina media, las paredes y membranas de la célula, participa en la división y extensión celulares, influye en la compartimentalización celular, modula la acción de hormonas y señales, y contribuye al equilibrio iónico de la célula (Marschner, 2002); además, participa en la disminución de la respiración del fruto y en el aumento de la fotosíntesis de la planta, procesos que en conjunto pueden aumentar la productividad de los cultivos. Resultados similares fueron obtenidos con la aplicación de nitrato de calcio en melón, pues se aumentó el número y el peso de los frutos por planta y el diámetro polar y ecuatorial del fruto (Román y Gutiérrez, 1998).

La producción favorable que fue obtenida con la aplicación de giberelinas, principalmente en calidad extra, se explica probablemente porque esta hormona aumenta la divisón y elongación celular y la entrada de agua a las células (Taiz y Zeiger, 2006), procesos que se traducen en aumento del tamaño final del fruto (Grange, 1996) y mayor acumulación de masa fresca, lo cual concuerda con los resultados obtenidos por Usenik et al. (2005), quienes demostraron que la aplicación de ácido giberélico en frutos de cereza aumentó el rendimiento y la calidad de estos. En uva también existen reportes de que las giberelinas aumentaron los rendimientos (Botelho et al., 2004; Vieira et al., 2008). Por el contrario, Paroussi et al. (2002) encontraron que al aplicar diferentes concentraciones de giberelinas en fresa, no se presentó un efecto positivo sobre el rendimiento.

\section{Rajado}

No se observaron diferencias estadísticas en el porcentaje de rajado para la calidad extra, sin em- bargo, todos los frutos con aplicaciones de calcio $y$ giberelinas mostraron un menor rajado que el testigo, principalmente con el tratamiento de 10 $\mathrm{mg} \mathrm{L}^{-1}$ de giberelinas. En la calidad I el rajado fue estadísticamente diferente, en este caso, en los frutos del testigo absoluto se presentó el menor porcentaje de rajado, mientras que en la calidad II la respuesta fue contraria pero sin diferencias estadísticas. El porcentaje de rajado en frutos de uchuva es más alto a medida que los frutos son más grandes (Fischer, 2005), razón por la cual, se presentó mayor rajado en la calidad extra, seguido de la calidad I y por último la calidad II. Para el porcentaje total de rajado, no hubo diferencias estadísticas, no obstante, con $10 \mathrm{mg} \mathrm{L}^{-1}$ de giberelinas el rajado de frutos fue menor pero se presentó mayor rajado con $1,5 \mathrm{~g} \mathrm{~L}^{-1}$ de calcio aún con más rajado que el testigo (figura 2).

Este último resultado no se esperaba, pues se ha mencionado que el calcio incrementa la firmeza del fruto al darle mayor estabilidad y rigidez a paredes y membranas celulares (Marschner, 2002) y esto aumenta la resistencia al rajado (Brown et al., 1996); sin embargo, la propiedad del calcio de reducir la extensibilidad de la pared en las dicotiledóneas (Taiz y Zeiger, 2006) podría ser un factor crítico relacionado con la sensibilidad al rajado, lo cual también fue encontrado en cerezas (Podestá et al., 2001). Es importante aclarar que este resultado desfavorable se debió a un alto porcentaje de rajado en las calidades I y II, y que las demás dosis de calcio sí lograron disminuir el rajado con relación al testigo.

Existe evidencia de que en frutos de Litchi chinensis el contenido de calcio está implicado en la resistencia al rajado, pues el pericarpio de frutos rajados tuvo significativamente más bajas concentraciones de calcio que frutos normales, esta situación se observó en el mismo árbol y aun en la misma panícula (Lin, 2001), lo cual se debe posiblemente a la diferencia en la actividad metabólica entre frutos y a las auxinas exportadas basipetalamente (Xu-Ming et al., 2008). 


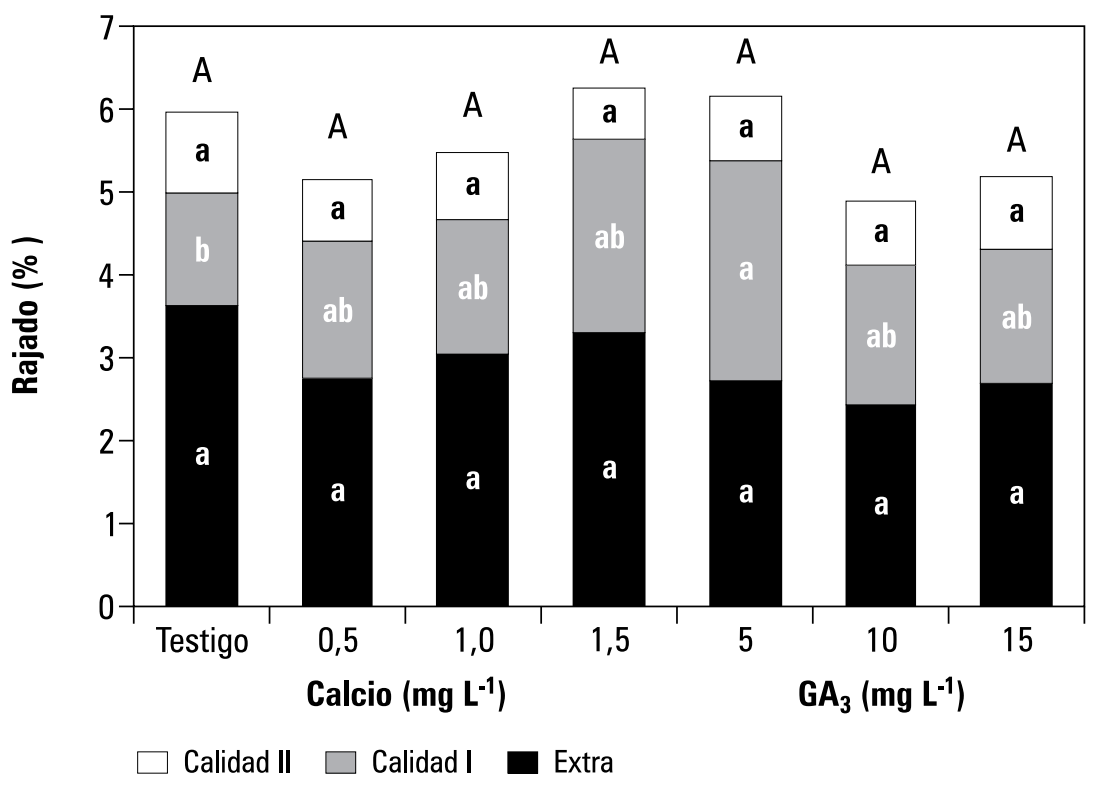

Figura 2. Efecto de las aplicaciones foliares de calcio y giberelinas en precosecha sobre el rajado de frutos de uchuva por calidades comerciales. Promedios con letras distintas en la misma serie indican diferencias significativas según la prueba de Tukey $(P \leq 0,05)$.

De acuerdo con Marschner (2002), el Ca permitiría una menor disgregación de los tejidos debido a que inhibe la síntesis de poligalacturonasa, responsable de la degradación de los pectatos de calcio. Según resultados experimentales obtenidos por Meheriuk et al. (1991) la aplicación foliar de $\mathrm{Ca}$ redujo hasta en un 75\% el rajado en cerezas. En concordancia, compuestos de calcio pulverizado aplicados durante la precosecha en cerezas pueden favorecer la firmeza de los frutos y su resistencia al rajado (Podestá et al., 1998).

Ben-Airie et al. (1986) afirman que la aplicación de giberelinas reduce el metabolismo respiratorio y la producción de etileno, lo que disminuye la síntesis de enzimas hidrolíticas. Al respecto, Andrews y Shulin (1995) encontraron que las giberelinas disminuyen la actividad de la poligalacturonasa y pectinmetilesterasa, enzimas hidrolíticas que se encuentran en la pared celulary que aumentan el rajado del fruto, lo cual podría explicar la disminución del rajado en los frutos de uchuva con la aplicación de giberelinas. Una alta firmeza producida por la aplicación de $10 \mathrm{mg} \mathrm{L}^{-1}$ de giberelinas podría expresar una modificación de la estructura de la pared celular que aumente la resistencia al rajado. Se ha sugerido que el aumento en la firmeza producida por las aplicaciones de giberelinas podría deberse a algún efecto del regulador de crecimiento sobre la estructura de la pared celular (Andrews y Shulin, 1995).

\section{Sólidos solubles totales}

La aplicación de los tratamientos presentó diferencias estadísticamente significativas en el contenido de SST, la dosis de $1 \mathrm{mg} \mathrm{L}^{-1}$ de calcio presentó valores de 16,2 y fue el tratamiento de menor contenido de azúcares, mientras que con $0,5 \mathrm{mg} \mathrm{L}^{-1}$ de calcio se obtuvo el valor más alto de SST, todos los valores presentados con la aplicación de giberelinas fueron inferiores al testigo 
(figura 3A). Los SST para todas los tratamientos se encuentran dentro de todos los rangos recomendados por la normatividad colombiana para uchuva fresca (Icontec, 1999).

Concentraciones altas de calcio en los tejidos del fruto resultan en una lenta maduración, baja tasa de respiración y retraso en la producción de etileno así como en el punto climatérico (Ernani et al., 2008), lo que indica que con los tratamientos de calcio (excepto con $0,5 \mathrm{mg} \mathrm{L}^{-1}$ ) posiblemente se retrasa la madurez de los frutos de uchuva, a su vez, la actividad enzimática encargada de la hidrólisis de polisacáridos de alto peso molecular a disacáridos y monosacáridos más simples (Taiz y Zeiger, 2006) también se reduce, por tanto, el contenido de SST es menor; lo cual también sería una probable explicación del efecto de las giberelinas, pues estas hormonas también retrasan la maduración (Facteau et al., 1992).

Otra posible explicación de la disminución de los SST podría ser que los tratamientos de $\mathrm{Ca}$ y giberelinas aumentaron el peso fresco de los frutos respecto al testigo, situación generada por un mayor contenido de agua en los tejidos, esto genera mayor dilución de todos los sólidos dentro del fruto y por ende menor contenido de SST.

Estos resultados difieren con investigaciones en donde se señala que existe un aumento en el contenido de SST en cerezas tratadas con giberelinas, y que este aumento es proporcional a la dosis aplicada (Facteau et al., 1985). Al respecto, Podestá et al. (2001) encontraron que en cerezas hubo mayor contenido de SST con aplicaciones de giberelinas y en este caso se debió a que los frutos tratados estuvieron más tiempo en el árbol y además el regulador de crecimiento pudo aumentar la tasa de acumulación de azúcares hacia los frutos. En fresa, Roussos et al. (2008) no encontraron efecto significativo de la aplicación de reguladores de crecimiento sobre los SST, lo cual coincide con lo encontrado por CasierraPosada y Salamanca (2008) en la misma especie bajo la aplicación precosecha de ácido giberélico y nitrato de calcio.

\section{Acidez total titulable}

Todos los tratamientos generaron un mayor contenido de ATT respecto al testigo absoluto $(P \leq 0,05)$ (figura 3B). Los ácidos orgánicos son, después
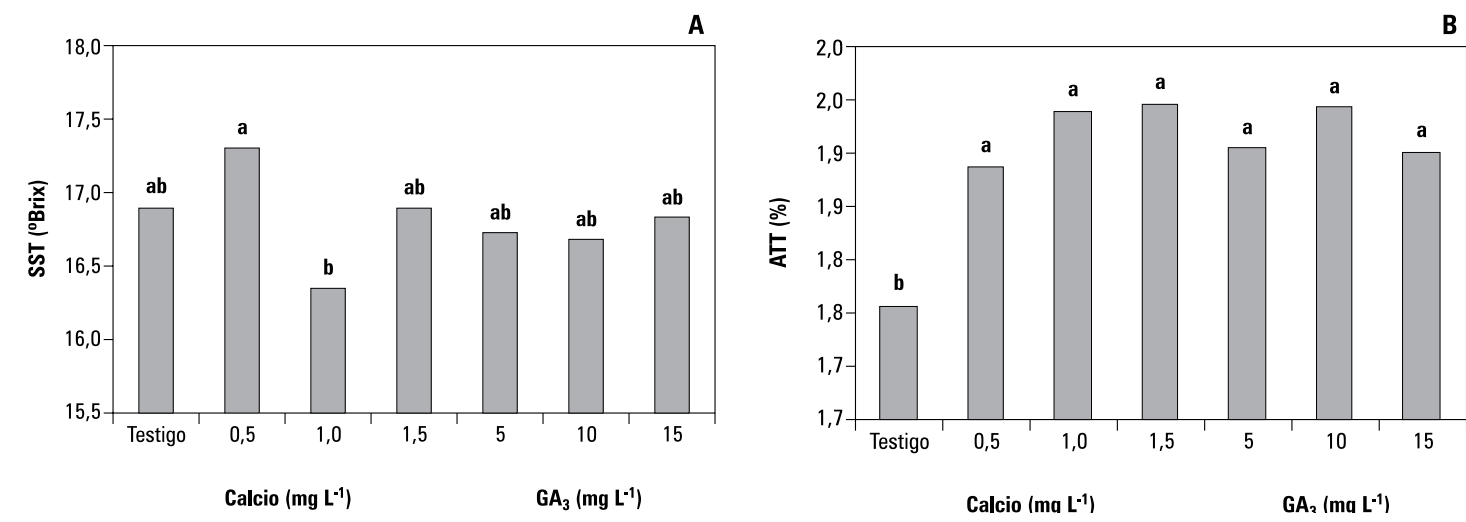

Figura 3. Efecto de las aplicaciones foliares de calcio y giberelinas en precosecha sobre los sólidos solubles totales (A) y la acidez total titulable (B) de frutos de uchuva. Promedios con letras distintas indican diferencias significativas según la prueba de Tukey $(P \leq 0,05)$. 
de los carbohidratos, el depósito energético más importante para el fruto (Osterloh et al., 1996). Durante la respiración del fruto son utilizados los ácidos orgánicos y convertidos a azucares, y varios de estos ácidos son componentes esenciales en el ciclo respiratorio de los ácidos tricarboxílicos (Kays, 2004). Esto indica que un mayor contenido de ácidos en la cosecha garantizaría una mayor vida útil en anaquel de los frutos de uchuva, por tanto, la aplicación de calcio y giberelinas favorecerían la duración poscosecha en uchuva. Una mayor vida útil en poscosecha con aplicaciones de ácido giberélico y nitrato de calcio fue encontrado en fresa, aunque sin diferencias estadísticas en la ATT (Casierra-Posada y Salamanca, 2008).

Aplicaciones de cloruro de calcio al 0,5\% en diferentes frecuencias $(0,4,8,12$ veces/año) a árboles de manzano no afectaron significativamente la calidad de los frutos respecto a la firmeza, SST, ATT, contenido de almidón y color (Ernani et al., 2008). Mientras que en cereza, un suministro foliar de calcio en precosecha aumentó la ATT, los SST y la relación de madurez (Podestá et al., 2001)

\section{Relación de madurez}

Para la RM se presentaron diferencias estadísticas entre los tratamientos, de tal forma que el testigo presentó el mayor valor de RM (figura 4A). Para el caso de la curuba Tellez et al. (2007), manifestaron que frutos con una alta RM tienden a ser mas agradables, pues una mayor RM indica un contenido predominante de azúcares sobre ácidos, lo que indica que los frutos del tratamiento testigo presentarían un mejor sabor, no obstante, una RM más baja con la aplicación de calcio y giberelinas en los frutos de uchuva posiblemente indica un retraso en la madurez que favorecería una mayor vida en poscosecha. Sin embargo, estos resultados fueron contrarios a los encontrados por Podestá et al. (2001) con aplicaciones precosecha de calcio y giberelinas en cereza, donde la RM fue mayor con estos tratamientos respecto al testigo.

\section{Firmeza}

La firmeza mostró diferencias significativas entre tratamientos, la mayor firmeza fue obtenida con la aplicación de $0,5 \mathrm{~g} \mathrm{~L}^{-1}$ de Ca. En general se encontró que tanto la aplicación de calcio como la de giberelinas presentó mayor firmeza que el testigo (excepto $5 \mathrm{mg} \mathrm{L}^{-1}$ de giberelinas) (figura 4B).

Las aplicaciones de calcio exógenas son efectivas para reducir el ablandamiento en todos los frutos (Saftner et al., 2003). Estas aplicaciones permiten que el calcio suministrado se una a las cargas negativas de los residuos de ácidos urónicos desesterificados que son generados por la enzima pectinmetilesterasa durante la maduración, aumentando la resistencia mecánica de los tejidos (Magee et al., 2003).

La aplicación de $3 \mathrm{~g} \mathrm{~kg}^{-1}$ de Ca en precosecha a frutos de Citrus sinenisis incrementó significativamente el contenido de Ca en la pulpa y corteza respecto a dosis menores (1 ó $2 \mathrm{~g} \mathrm{~kg}^{-1}$ de Ca), sin embargo, con cualquiera de las aplicaciones se disminuyó la actividad de poligalacturonasa, pectinesterasa y B-galactosidasa, mediante la influencia de la expresión de genes, y por tanto, la disolución e hidrólisis de pectinas fueron restringidas. También modificaron la estructura de los tejidos de la membrana, siendo estos más compactos y homogéneos con mayor presencia de Ca (Dong et al., 2009).

El aumento de la firmeza por aplicaciones de giberelinas podría deberse a algún efecto del regulador de crecimiento sobre la estructura de la pared celular (Andrews y Shulin, 1995), o simplemente a un retraso en la maduración de los frutos, que permitiría mantener la integridad de las membranas y paredes celulares por más tiempo al inhibir la acción de enzimas hidrolíticas que actúan sobre la pectina. Una mayor firmeza y un retraso en la maduración por acción de las giberelinas fue encontrado por Facteau et al. (1992). 

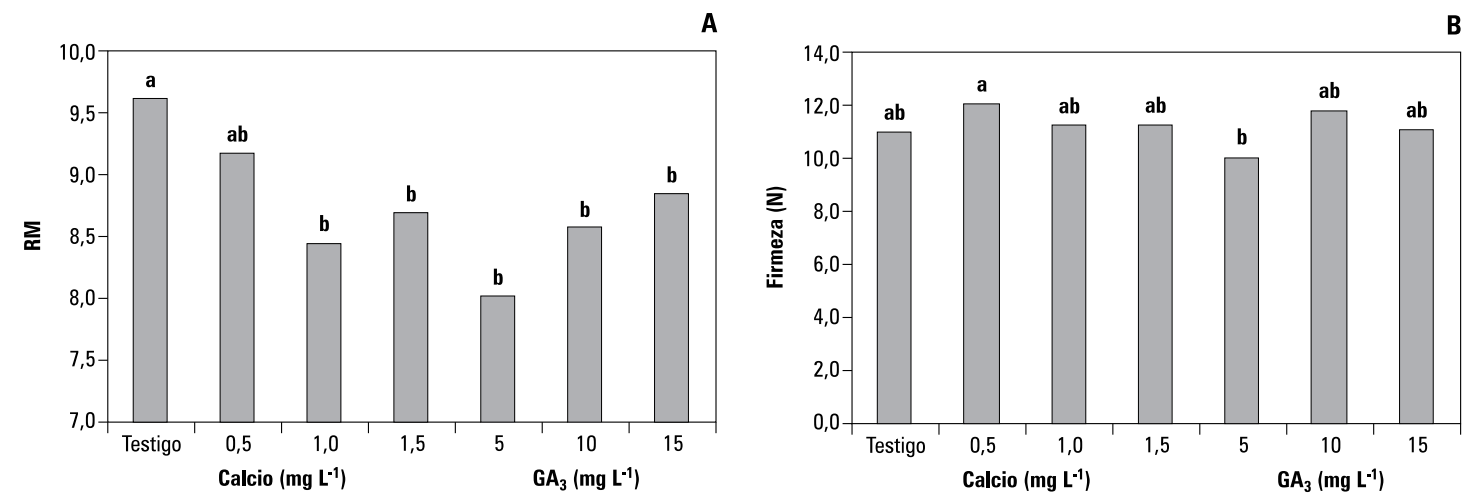

Figura 4. Efecto de las aplicaciones foliares de calcio y giberelinas en precosecha sobre la relación de madurez (RM) (A) y la firmeza (B) de frutos de uchuva. Promedios con letras distintas indican diferencias significativas según la prueba de Tukey $(P \leq 0,05)$.

\section{CONCLUSIONES}

Bajo las condiciones del experimento, la aplicación de calcio y giberelinas favoreció la producción de frutos de uchuva y disminuyó el porcentaje de rajado de los mismos, especialmente en concentraciones de 0,5 y $1,0 \mathrm{mg} \mathrm{L}^{-1}$ de calcio y 10 y $15 \mathrm{mg} \mathrm{L}^{-1}$ de $\mathrm{GA}_{3}$. Además, estos tratamientos presentaron un incremento en la firmeza y ATT y una disminución en los SST y la RM, lo cual indica que retrasan la madurez y pueden conservar la calidad de los frutos por más tiempo.

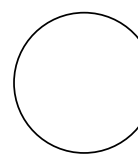

AGRADECIMIENTOS

Los autores agradecemos al ingeniero agrónomo Alfonso Molano, propietario de la finca El Saque, por su valiosa colaboración para que la presente investigación llegara a buen término. 


\section{REFERENCIAS BIBLIOGRÁFICAS}

Agronet. 2008. Producción y área cosechada de uchuva en Colombia en 2008. Ministerio de Agricultura y Desarrollo Rural. En: http://www.agronet.gov.co/; consulta: diciembre de 2008.

Andrews, P.K. y L. Shulin. 1995. Cell wall hydrolytic enzyme activity during development of nonclimacteric sweet cherry (Prunus avium L.) fruit. J. Hort. Sci. 70 (4), 561-567.

Barker, A. y D. Pilbeam. 2007. Handbook of plant nutrition. CRC Press Taylor \& Francis Group, New York, NY.

Ben-Airie, R.; H. Bazak y A. Blumenfeld. 1986. Gibberellin delays harvest and prolongs life of persimmon fruits. Acta Hort. 179, 807-813.

Botelho, R.V.; E.J.P. Pires y M.M. Terra. 2004. Efeitos de reguladores vegetais na qualidade de uvas 'Niagara Rosada' na região noroeste do Estado de São Paulo. Rev. Bras. Frutic. 26(1), 74-77.

Brown, G.S.; S. Wilson; W. Boucher; B. Graham y B. McGlasson. 1996. Effects of copper-calcium sprays on fruit cracking in sweet cherry (Prunus avium). Scientia Hort. 62, 75-80.

Casierra-Posada, F. y R. Salamanca. 2008. Influencia del ácido giberélico y del nitrato de calcio sobre la duración poscosecha de frutos de fresa (Fragaria sp.). Rev. Colomb. Cienc. Hortic. 2(1), 33-42.

Cooman, A.; C. Torres y G. Fischer. 2005. Determinación de las causas del rajado del fruto de uchuva (Physalis peruviana L.) bajo cubierta: II. Efecto de la oferta de calcio, boro y cobre. Agron. Colomb. 23(1), 74-82.

Dong, T.; R. Xia; Z. Xiao; P. Wanga y W. Song. 2009. Effect of pre-harvest application of calcium and boron on dietary fibre, hydrolases and ultrastructure in 'Cara Cara' navel orange (Citrus sinensis L. Osbeck) fruit. Scientia Hort. 121 (en imprenta).

Ernani, P.R.; J. Dias; C.V.T. Do Amarante; D.C. Ribeiro y D.A. Rogeri. 2008. Preharvest calcium sprays were not always needed to improve fruit quality of 'Gala' apples in Brazil. Rev. Bras. Frutic. 30(4), 892-896.

Facteau, T.J.; N.E. Chestnut; K.E. Rowe y C. Payne. 1992. Brine quality of gibberellic acid-treated "Napoleon" sweet cherries. HortScience 27(2), 118-122.
Facteau, T.J.; K.E. Rowe y N.E. Chestnut. 1985. Response patterns of gibberellic acid-treated sweet cherry fruit at different soluble solids levels and leaf/fruit ratios. Scientia Hort. 27, 257-262.

Gordillo, O.; G. Fischer y R. Guerrero. 2004. Efecto del riego y de la fertilización sobre la incidencia del rajado en frutos de uchuva (Physalis peruviana L.) en la zona de Silvania (Cundinamarca). Agron. Colomb. 22 (1), $53-62$.

Grange, R. 1996. Crecimiento del fruto. pp. 449-462. En: Azcón-Bieto, J. y M.Talón (eds.). Fisiología y bioquímica vegetal. Interamericana-McGraw-Hill, Madrid.

Fischer, G. 2005. El problema del rajado del fruto de uchuva y su posible control. pp. 55-82. En: Fischer, G., D. Miranda, W. Piedrahita y J. Romero (eds.) Avances en cultivo, poscosecha y exportacion de la uchuva (Physalis peruviana L.) en Colombia. Facultad de Agronomia, Universidad Nacional de Colombia; Unibiblos, Bogotá.

Hernández-Muñoz, P.; E. Almenar; V. Del Valle; D. Velez y R. Gavara. 2008. Effect of chitosan coating combined with postharvest calcium treatment on strawberry (Fragaria $x$ ananassa) quality during refrigerated storage. Food Chem. 110, 428-435.

Huang, X.M.; H.C. Wang; J.G. Li; J.H. Yin; W.Q. Yuan; J.M. Lu y H.B. Huang. 2005. An overview of calcium's role in lychee fruit cracking. Acta Hort. 665, 231-240.

Icontec. 1999. Frutas frescas. Uchuva. Especificaciones. Norma Técnica Colombiana NTC 4580. Instituto Colombiano de Normas Técnicas y Certificación, Bogotá.

Kays, S. 2004. Postharvest biology. Exon Press, Athens.

Lin, L.W. 2001. Effect of mineral nutrient on fruit cracking rate of Litchi chinensis Sonn. Soil Environ. Sci. $10,55-56$

Looney, N.E. y P.D. Lidster. 1980. Some growth regulator effects on fruit quality, mesocarp composition, and susceptibility to postharvest surface marking of sweet cherries. J. Amer. Soc. Hort. Sci. 105(1), 130-134

Magee, R.L.; F. Caporaso y A. Prakash. 2003. Effects of exogenous calcium salt treatments on inhibiting 
irradiation-induced softening in diced roma tomatoes. J. Food Sci. 68, 2430-2435.

Marschner, H. 2002. Mineral nutrition of higher plants. $2 \mathrm{a}$ ed. Academic Press, London.

Matsuoka, M. 2003. Gibberellins signaling: how do plant cells respond to GA signals? Plant Growth Regul. 22, 123-125.

Meheriuk, M.; G.H. Neilsen y D.L. Mckenzie. 1991. Incidence of rain splitting in sweet cherries treated with calcium or coating materials. Can. J. Plant Sci. 71, 23 1-234.

Opara, L. 1997. Fruit skin splitting and cracking. Hort. Rev. 19, 217-262

Osterloh, A.; G. Ebert; W. Held; H. Schulz y E. Urban. 1996. Lagerung von Obst und Südfrüchten. Verlag Ulmer, Stuttgart, Alemania.

Paroussi, G.; D.G. Voyiatzis; E. Paroussi y P.D. Drogour. 2002. Growth, flowering and yield responses to $\mathrm{GA}_{3}$ of strawberry grown under different environmental conditions. Scientia Hort. 9,103-113.

Peet, M.M. 1992. Fruit cracking in tomato. Hort Technol. 2, 216-223

Podestá, L.; F. Gil; M. Rodríguez y C. Arjona. 2001. Efecto del ácido giberélico y del calcio sobre el tamaño, agrietamiento y otros parámetros de calidad en frutos de cerezo (Prunus avium L.) cv. Bing. Invest. Agr. Prod. Prot. Veg. 16 (1), 37-48.

Podestá, L.; F. Gil; M. Rodríguez y C. Arjona. 1998. Efecto del calcio y el ácido giberélico sobre el tamaño, el cracking y otros parámetros de calidad en frutos de cerezo (Prunus avium L.) cv. Bing. XXI Congreso Argentino de Horticultura. Departamento de Producción Agropecuaria, Universidad Nacional de Cuyo, Mendoza, Argentina.

Pratella G.C. 1996. La screpolatura delle ciliege. Rivista di Frutticoltura e di Ortofloricoltura 10, 71-73.

Román, L.F. y M.A. Gutiérrez. 1998. Evaluación de ácidos carboxílicos y nitrato de calcio para incremen- tar la calidad, cantidad y vida en anaquel en tres tipos de melón. Terra 16(1), 49-54.

Roussos, P.A.; N.K. Denaza y T. Damvakaris. 2008. Strawberry fruit quality attributes alter application of plant growth stimulating compounds. Scientia Hort. 119(2), 138-146.

Saftner, R.A.; J. Bai; J.A. Abbott y Y.S. Lee. 2003. Sanitary dips with calcium propionate, calcium chloride, or calcium amino acid chelates maintain quality and shelf stability of fresh-cut honeydew. Postharv. Biol. Technol. 29, 257-269.

Sansavini, S. y S. Lugli. 1997. Prospettive e innovazioni tecnico-scientifiche per la cerasicoltura italiana. Rivista di Frutticoltura e di Ortofloricoltura 6, 918.

Sekse, L. 1995. Cuticular fracturing in fruit cherry (Prunus avium L.) resulting from changing soil water contents. J. Hort. Sci. 70(4), 631-635.

Taiz L. y E. Zeiger. 2006. Plant physiology. 4a ed. Sinauer Associates Publishers, Sunderland, MA.

Téllez, C.P.; G. Fischer y O. Quintero. 2007. Comportamiento fisiológico y fisicoquímico de frutos de curuba (Passiflora mollissima Bailey) encerados y almacenados a dos temperaturas. Rev. Colomb. Cienc. Hort. 1(1), 67-80.

Usenik, V.; D. Kastelec y F. Tampar. 2005. Physicochemical changes of sweet cherry fruits related to application of gibberellic acid. Food Chem. 90(4), 663-671.

Valencia, M. 1985. Anatomía del fruto de la uchuva. Acta Biol. Colomb. 1(2), 63-89.

Vieira, C.R.Y.; E.J. Pires; M.M. Terra; M.A. Tecchio y R.V. Botelho. 2008. Efeitos do ácido giberélico e do thidiazuron sobre as características dos frutos e do mosto da uva 'Niagara Rosada'. Rev. Bras. Frutic. $30(1), 12-19$.

Xu-Ming, H.; W. Hui-Cong; Z. Wei-Liang; Y. Wei-Qun; L. Jie-Mei y L. Jian-Guo. 2008. Spraying calcium is not an effective way to increase structural calcium in litchi pericarp. Scientia Hort. 117, 39-44. 